\title{
The effect of induced hypotension on the perioperative bleeding and transfusion in the bipolar hemiarthroplasty of hip: retrospective study for four years
}

\author{
Sae-Cheol Oh, Seung Uk Bang, and Byung-Gil Kang \\ Department of Anesthesiology and Pain Medicine, College of Medicine, The Catholic University of Korea, Daejeon, Korea
}

It is well known that induced hypotensive anesthesia results in reduced blood loss and allows for a cleaner surgical field intraoperatively, leading to improved visualization. We investigated the effect of induced hypotensive anesthesia compared to that of normotensive anesthesia on blood loss and found that induced hypotension can reduce the volume of transfusion during the hospitalization period in bipolar hemiarthroplasty (BPHA) of the hip.

We retrospectively reviewed the anesthetic records, nursing notes, and hematologic laboratory data. We divided the patients into two groups, with patients who underwent surgery from January 2008 to December 2009 placed in the control group, while patients who received surgery between January 2011 and July 2012 were placed in the experimental group. We excluded patients who received surgery in the year of 2010 to diminish bias because we did not have a standardized protocol for induced hypotension anesthesia during that year. In addition, all operations were performed by a single orthopedic hip expert who had been operating for twenty-five years.

Patients in both groups received three types of anesthesia: spinal, epidural, and general anesthesia. Normotensive anesthesia was administered with the maintenance of mean arterial pressure (MAP) between 70-80 $\mathrm{mmHg}$ without considering intraoperative induced hypotension. In the experimental group, however, anesthesia was administered by a single orthopedic anesthesia specialist according to his protocol for induced hypotension. The goal of induced hypotension was maintenance of
MAP between 55-60 mmHg. When an adjunct was necessary, nicardipine $2-5 \mathrm{mg} / \mathrm{hr}$ was infused continuously. Values of MAP $<55 \mathrm{mmHg}$ were treated with intravenous fluids, ephedrine, and phenylephrine therapy.

We collected data on operation time, blood loss, Hemoglobin $(\mathrm{Hb})$, Hematocrit (Hct), and transfusions. Intraoperative blood loss was measured by the anesthesiologist based on the contents of suction bottles and the increase in weight of surgical swabs. Blood loss in the postoperative period was estimated from the volume in the drainage bags. Postoperative $\mathrm{Hb}$ and Hct were measured three days after the surgery. SPSS 14.0 was used for statistical analysis (SPSS Inc. Released 2006. SPSS for Windows, Version 14.0. Chicago, IL, USA). Continuous values were analyzed using the Z-test, and binary values using the chi-square test. Null hypotheses were rejected if $\mathrm{P}$ values were less than 0.05 . Demographic and perioperative data for the two groups are shown in Table 1.

Many studies have been performed which measured intraoperative blood loss in total hip replacement arthroplasty (THRA), but there has been no specific study to date which measured the blood loss in BPHA. The effect of an intervention designed to reduce blood loss would be expected to be similar in BPHA and in THRA, however, as the two procedures are not significantly different. Among patients undergoing THRA, the group with induced hypotension experienced a blood loss volume during surgery of approximately 300-400 ml, which was significantly lower than the blood loss volume during surgery for the group

Corresponding author: Seung Uk Bang, M.D., Department of Anesthesiology and Pain Medicine, College of Medicine, The Catholic University of Korea, 520-2, Daeheung-dong, Jung-gu, Daejeon 301-723, Korea. Tel: 82-42-242-9046, Fax: 82-42-242-3500, E-mail: seungukb@naver.com (c) This is an open-access article distributed under the terms of the Creative Commons Attribution Non-Commercial License (http:// creativecommons.org/licenses/by-nc/3.0/), which permits unrestricted non-commercial use, distribution, and reproduction in any medium, provided the original work is properly cited. 
Table 1. Demographic Data and Operative Data

\begin{tabular}{lcc}
\hline & $\begin{array}{c}\text { Control group } \\
(\mathrm{n}=79)\end{array}$ & $\begin{array}{c}\text { Induced } \\
\text { hypotension group } \\
(\mathrm{n}=85)\end{array}$ \\
\hline Age (yr) & $76.8 \pm 9.7$ & $80 \pm 7.2^{*}$ \\
Sex $(\mathrm{M} / \mathrm{F})$ & $17 / 62$ & $13 / 72$ \\
Weight $(\mathrm{kg})$ & $50.8 \pm 11$ & $49.8 \pm 10.2$ \\
Type of anesthesia (n) & & \\
$\quad$ General anesthesia & 33 & $12^{*}$ \\
$\quad$ Epidural anesthesia & 3 & 3 \\
$\quad$ Spinal anesthesia & 43 & $70^{*}$ \\
Duration of operation (min) & $51 \pm 35.8$ & $55.1 \pm 18.2$ \\
Blood loss (ml) & & \\
$\quad$ Intraoperative loss & $409.4 \pm 173.5$ & $295.7 \pm 158.9^{*}$ \\
$\quad$ Postoperative loss & $324.3 \pm 223.5$ & $378.9 \pm 257.9$ \\
Hemoglobin (g/dl) & & \\
$\quad$ Before operation & $12.2 \pm 1.6$ & $11.7 \pm 1.3^{*}$ \\
$\quad$ After operation & $11.2 \pm 1.1$ & $11.3 \pm 1.4$ \\
Hematocrit (\%) & & \\
$\quad$ Before operation & $36.2 \pm 4.5$ & $35 \pm 3.6^{*}$ \\
$\quad$ After operation & $34.4 \pm 3.5$ & $34.9 \pm 4.6$ \\
PRBCs transfusion (units) & & \\
$\quad$ Intraoperative & $0.45 \pm 0.76$ & $0.3 \pm 0.5$ \\
$\quad$ Postoperative & $1.9 \pm 1.5$ & $2.6 \pm 1.7^{*}$ \\
$\quad$ Neurologic complication & None & None \\
\hline Postopertive &
\end{tabular}

Postoperative $\mathrm{Hb}$ and Hct measured three days later from surgery. Data are means \pm SD. PRBC: packed red blood cell. $* \mathrm{P}<0.05$ compared to control group.

without induced hypotension [1-3]. Our hospital measured a reduction in blood loss volume of about $28 \%$ during surgery, which is similar to previous reports. However, contrary to our expectations, the volume of blood transfusion after surgery was larger in the induced hypotension group, despite the smaller volume of blood loss during the intraoperative period. To analyze these results, we focused on the age of patients and their preoperative levels of $\mathrm{Hb}$ and $\mathrm{Hct}$.

First, the mean age of patients in the induced hypotension group was 80 years, while that of patients in the control group was 76.8 years. In addition, the induced hypotension group had significantly lower preoperative levels of $\mathrm{Hb}$ and Hct than the control group. We considered age as a major contributing factor to the patients' $\mathrm{Hb}$ and Hct levels. That is to say, the older the patients, the lower their levels of $\mathrm{Hb}$ and Hct. Zakai et al. [4] reported that $\mathrm{Hb}$ decreases with increasing age; as a result, there is an increased incidence of anemia with age. Furthermore, Salive et al. [5] reported that $\mathrm{Hb}$ and anemia were related to factors such as age, race, body mass index, smoking, cancer, and renal insufficiency; among these factors, the strongest connection was with age.

In the current study, the induced hypotension group, which had a higher average age than the control group, experienced approximately $28 \%$ less blood loss volume during surgery. However, there was no difference in blood transfusion volume during surgery between the two groups. Although there is a reduction of $28 \%$ in blood loss volume through the induced hypotension technique, it amounts to only $114 \mathrm{ml}$ if we calculate the volume of blood saved from the total amount of bleeding in the control group. This amount is not sufficient to have a significant influence on the volume of transfusion during surgery. The decreased blood loss is also not sufficient to compensate for the differences in $\mathrm{Hb}$ and $\mathrm{Hct}$ which were observed between the two groups before the surgery. In addition, the transfusions after surgery were conducted by the orthopedic surgeon, who performed transfusions in cases of $\mathrm{Hb}$ below $10 \mathrm{~g} / \mathrm{dl}$. Hence, the volume of blood transfusion was larger in the induced hypotension group compared to the control group because the induced hypotension group had relatively lower $\mathrm{Hb}$ and Hct before surgery.

There were also differences between the two groups in the type of anesthesia administered. Kim et al. [3] reported that forty-seven patients scheduled for THR were sorted into three groups: epidural anesthesia, combined general epidural anesthesia, and general anesthesia. They compared intraoperative blood loss in the three groups under the same MAP $(60 \pm 5 \mathrm{mmHg})$ and observed no differences in blood loss among the three groups. Therefore, we can conclude that differences of anesthetic type in our study had little effect on any major differences in intraoperative blood loss.

Currently, it is widely used in orthopedic surgery in order to reduce the volume of blood transfusion and prevent edema in the surgical area. Based on the results of our hospital's retrospective study, although induced hypotension does not reduce the volume of transfusion during the hospitalization period, it can be concluded that applying induced hypotensive anesthesia during BPHA can reduce blood loss volume during surgery. A future prospective study should evaluate this issue.

\section{References}

1. Sharrock NE, Mineo R, Urquhart B, Salvati EA. The effect of two levels of hypotension on intraoperative blood loss during total hip arthroplasty performed under lumbar epidural anesthesia. Anesth Analg 1993; 76: 580-4.

2. Niemi TT, Pitkänen M, Syrjälä M, Rosenberg PH. Comparison of hypotensive epidural anaesthesia and spinal anaesthesia on blood loss and coagulation during and after total hip arthroplasty. Acta Anaesthesiol Scand 2000; 44: 457-64. 
3. Kim JM, Hong JH, Jang YH, Lee YC, Kwon SH. Comparison of blood loss according to different anesthetic technique for total hip replacement. Korean J Anesthesiol 2007; 53: 583-8.

4. Zakai NA, Katz R, Hirsch C, Shlipak MG, Chaves PH, Newman AB, et al. Prospective study of anemia status, hemoglobin concentration, and mortality in an elderly cohort: the Cardiovascular Health Study. Arch Intern Med 2005; 165: 2214-20.

5. Salive ME, Cornoni-Huntley J, Guralnik JM, Phillips CL, Wallace RB, Ostfeld AM, et al. Anemia and hemoglobin levels in older persons: relationship with age, gender, and health status. J Am Geriatr Soc 1992; 40: 489-96. 